\title{
Galactose- $\alpha-1,3$-galactose allergy: a rare syndrome and an atypical presentation
}

\author{
${ }^{1}$ Department of Immunoallergology, Hospital de Santo António, Centro Hospital do Porto, Oporto, Portugal
}

${ }^{2}$ Research Department of BIAL Arístegui Research and Development, Bilbao, Spain

\section{KEY WORDS}

allergy; anaphylaxis; galactose- $\alpha$ 1,3-galactose; red meat; SDS-PAGE IgE-immunoblotting

\section{Corresponding author}

Carmo Abreu

Departamento de Imunoalergologia

Centro Hospitalar do Porto

Largo Prof. Abel Salazar

4099-001 Porto, Portugal

Phone: +351222077500

Fax: +351222077 503

E-mail: nuchabreu@gmail.com

\section{Doi}

10.23822/EurAnnACI.1764-1489.49

\begin{abstract}
Summary
Allergies to red meat associated with galactose- $\alpha-1,3$-galactose, commonly known as $\alpha$-gal, are rare and have only recently been described. At this time, the literature reports only one case documented in Portugal. In this study, we report the case of a 76-year-old male with an immediate reaction following the ingestion of red meat. Rigorous diagnostic exams, including prick test, prick-to-prick tests, serum specific IgE and SDS-PAGE IgE-immunoblotting, were performed. The $\alpha$-gal epitope IgE returned a value of $35.3 \mathrm{kUA} / \mathrm{L}$, leading the authors to believe that this is an atypical case of $\alpha$-gal allergy.
\end{abstract}

\section{Introduction}

Recent studies have shown that mammalian meat allergy is an emergent allergy worldwide. Many cases of delayed anaphylaxis to red meat have been described, especially in the United States (1). The basis for these reactions appears to be the presence of specific immunoglobulin $\mathrm{E}$ ( $\mathrm{IgE}$ ) antibodies against the oligosaccharide $\alpha$-gal, a carbohydrate in non-primate mammals that does not exist in humans. Exposure to this oligosaccharide occurs with the ingestion of meat, offal and gelatin with an origin in beef, pork, lamb, horse and deer. This epitope may also be present in drugs, vaccines, and dairy products.

The typical presentation of $\alpha$-gal allergy involves a delayed onset reaction, i.e., occurring 4 to 8 hours after the consumption of mammalian meat products (2). The symptoms usually in- clude urticaria and angioedema, and can progress to potentially fatal anaphylaxis.

The $\alpha$-gal hypersensitivity has been reported in an association between an episode of tick bite and the subsequent development of symptoms in response to the ingestion of red meat. Reactions to cetuximab, a monoclonal antibody against EGFR (epidermal growth factor receptor), occur immediately after the first administration due to the presence of the epitope $\alpha$-gal $(3,4)$.

\section{Case Report}

Herein, we report the case of a 76-year-old Caucasian male who experienced an episode of anaphylaxis (sickness, diarrhea, vomiting and cutaneous lesions scattered over the body) $1 \mathrm{~h}$ after a meal consisting only of rice and beef. After self-medication with 
oral corticosteroid and antihistamines, the reaction resolved completely within a few hours, and without any residual damage. Since then, the patient has self-excluded this specific type of food from his diet.

Two months later and following a continuous period of abstinence from eating red meat, the patient unintentionally ingested a blend of cooked meats (beef, pork, and sausages) and immediately experienced anaphylactic shock (diarrhea, vomiting, cutaneous lesions, hypotension and syncope). Upon admission to the emergency department, the patient was treated with antihistamines, epinephrine, corticosteroids and intravenous fluid therapy, with a full resolution of his symptoms. The patient was referred to the immunoallergy unit.

The patient presented no family history of atopy or allergic disorders and no personal comorbidities such as asthma or other allergies. Neither of the anaphylactic episodes was preceded by physical exercise or ingestion of any kind of drug, and the patient denied having been stung or bitten by an insect.

In the allergy unit, the patient underwent a diagnostic workup including skin-prick testing with a panel of aeroallergens and food allergens (Dermatophagoides pteronyssinus, cat and dog epithelia extracts, grass, cow's milk, egg, wheat and fish (Bioportugal ${ }^{\circledR}$, ALK-Abelló, Madrid, Spain). The tests returned positive results for cow's milk and for cat and dog epithelia extracts. Prick-toprick tests were also performed for raw and cooked pork and beef, returning positive results for raw pork and cooked beef.

Laboratory tests revealed an elevated serum total IgE value by ImmunoCAP ${ }^{\circledR}$ (Thermo Fisher Scientific, Phadia, US) of 559 $\mathrm{kUA} / \mathrm{L}$. Serum-specific IgE values were also determined for cat epithelia (4.82 kUA/L), dog epithelia (1.04 kUA/L), cow's milk (2.72 kUA/L), beef $(8.6 \mathrm{kUA} / \mathrm{L})$ and pork $(6.67 \mathrm{kUA} / \mathrm{L})$.

Due to the IgE value for cow's milk, together with several non-

Figure 1 - SDS-PAGE IgE-immunoblotting. A, milk extract; $B$, beef (raw); $C$, beef extract (raw); $D$, pork (raw); E, pork extract (raw); F, BSA (bovine serum albumin); G, cat epithelium extract; $H$, dog epithelium extract; $I$, dog dander extract; $J$, cat serum albumin; $P$, patient serum; $C$, serum from negative control group; $M$, standard molecular masses.

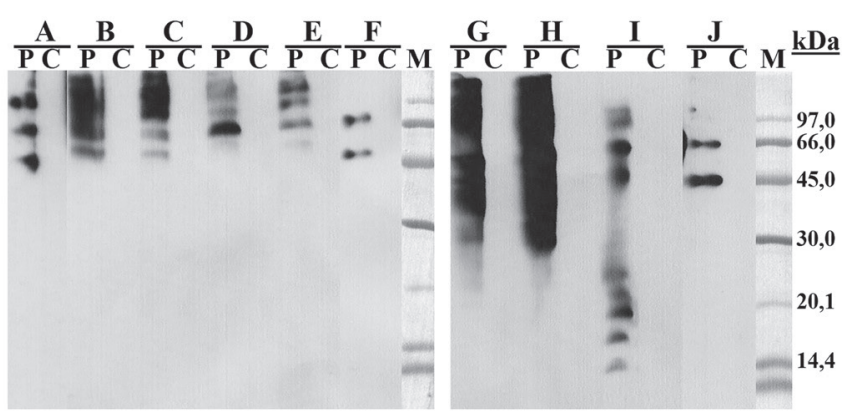

specific gastrointestinal complaints associated with milk ingestion, we conducted an oral provocation test with milk that was negative (cumulative dose of $200 \mathrm{ml}$ ).

With the collaboration of the research department of BIAL Arístegui, SDS-PAGE IgE-immunoblotting with pork, beef, bovine serum albumin (BSA), cat and dog epithelia was performed.

As presented in figure 1, beef and pork IgE binding were detected in the bands of the same molecular mass: batch of molecular mass $>45 \mathrm{kDa}$. Regarding extracts from cat and dog epithelia, IgE binding is also observed in bands of molecular mass $>45$ $\mathrm{kDa}$; in the dog epithelia extract, $\mathrm{IgE}$ binding is observed in bands of approximately $67 \mathrm{kDa}$ and $45 \mathrm{kDa}$. Bovine serum albumin (BSA) also had the same molecular mass for the bands of approximately 67 and $45 \mathrm{kDa}$ (figure $\mathbf{1}$ ).

IgE binding bands of the same molecular mass as mammalian serum albumin (BSA and cat albumin) have been detected (approximately 67 e $45 \mathrm{kDa}$ ). An important differential diagnosis with $\alpha$-gal allergy is the pork syndrome, since they have several characteristics in common. Both involve IgE-mediated reactions triggered by the ingestion of mammalian meat. Both syndromes may show similar results in skin-prick tests and immunoassay by cross-reactivity. Patients with allergy to red meat from hypersensitivity to $\alpha$-gal have high specific $\operatorname{IgE}$ levels for beef, pork, lamb, cat epithelia, dog epithelia and cow's milk. These patients have high specific IgE to cat epithelia, due to the $\alpha$-gal residues present in cats' $\operatorname{IgE}$ and not due to a positivity of Fel d 1, the cat's main allergen (5).

Specific IgE against cat serum albumin was determined with values of $<0.35 \mathrm{kUA} / \mathrm{L}$. Finally, an $\alpha$-gal epitope $\mathrm{IgE}$ was performed, returning a value of $35.3 \mathrm{kUA} / \mathrm{L}$. The levels of $\alpha$-gal were calculated by the ImmunoCAP method (Thermo Fisher, Vitoria, Basque Country).

The study was conducted in accordance with the ethical standards established in the Declaration of Helsinki of 1946, and that informed consent was obtained from the patient before enrolment in the study.

\section{Conclusions}

The major allergens involved in allergic reactions to mammalian red meat are serum albumin and immunoglobulins. In these patients, it may be difficult to identify a cause for the reactions, especially if there is no history of tick bite or exposure to cetuximab. Therefore, skin tests have limited value for diagnosis, making IgE for specific $\alpha$-gal essential for diagnosis. We report an unusual case of immediate reaction to meat, with the detection of high serum specific IgE values to $\alpha$-gal, in a patient with no history of tick bite or exposure to intravenous cetuximab. The patient has now been avoiding red meat for two years and has had no further reactions. 


\section{Conflict of interest}

The authors declare that they have no conflict of interest.

\section{References}

1. Commins SP, Platts-Mills TA. Anaphylaxis syndromes related to a new mammalian cross-reactive carbohydrate determinant. J Allergy Clin Immunol 2009; 124(4):652-7.

2. Commins SP, Satinover SM, Hosen J, Mozena J, Borish L, Lewis $\mathrm{BD}$, et al. Delayed anaphylaxis, angioedema, or urticaria after consumption of red meat in patients with $\mathrm{IgE}$ antibodies specific for galactose-alpha-1,3-galactose. J Allergy Clin Immunol 2009; 123(2):426-33.

3. Van Nunen SA, O'Connor KS, Clarke LR, Boyle RX, Fernando SL. An association between tick bite reactions and red meat allergy in humans. Med J Aust 2009; 190(9):510-1.

4. Jacquenet S, Moneret-Vautrin DA, Bihain BE. Mammalian meat-induced anaphylaxis: clinical relevance of anti-galactose-alpha-1,3-galactose IgE confirmed by means of skin tests to cetuximab. J Allergy Clin Immunol 2009; 124(3):603-5.

5. Steinke JW, Platts-Mills TA, Commins SP. The alpha-gal story: lessons learned from connecting the dots. J Allergy Clin Immunol 2015; 135(3):589-96. 